\title{
APROVEITAMENTO DO SILÍCIO DERIVADO DA EXTRAÇÃO DO QUARTZITO SÃO THOMÉ NA CULTURA DO PEPINO
}

\author{
Eliana Alcantra \\ Doutora em Entomologia. Docente no Mestrado Sustentabilidade em Recursos Hídricos e no \\ curso de Agronomia na Universidade Vale do Rio Verde, Três Corações - MG \\ lialcantra@yahoo.com.br
}

Marco Antônio Sales Martins Bacharel em Agronomia Graduando do curso de Agronomia da Universidade Vale do Rio Verde-Unincor, Três Corações - MG. marcomartinspedras@hotmail.com

Fredderico Avelar Viana Corrêa Bacharel em Agronomia da Universidade Vale do Rio Verde-Unincor, Três Corações - MG. favc92@gmail.com

\section{Rosângela Francisca de Paula Vitor Marques} Doutora em Recursos Hídricos. Docente da Universidade Vale do Rio Verde nos cursos de Mestrado em Sustentabilidade em Recursos Hídricos, Engenharia Ambiental e Sanitária e

Agronomia, Três Corações, MG. rosangela.marques@unincor.edu.br

\section{RESUMO}

O silício absorvido pelas plantas melhora a sua morfologia e fisiologia, com redução na taxa de transpiração e confere eficiência fotossintética às plantas, é muito eficiente na proteção de estresses abióticos e bióticos de muitas culturas e pode conferir resistência ao ataque de insetos-praga e nematóides. O silício derivado da extração e produção mineral de quartzito em São Thomé das Letras, MG tem se tornado um rejeito gerador de problema ambiental e o seu aproveitamento na agricultura pode ser uma forma de uso sustentável. Assim, objetivouse com este trabalho avaliar o uso do silício, derivado da extração e produção mineral de quartzito São Thomé, na cultura do pepino. O experimento foi implantado na Fazenda São Francisco de Assis, em Três Corações - MG. O delineamento foi em blocos casualizados com dois tratamentos e 10 repetições. Os tratamentos utilizados foram: T1: 3 ton.ha ${ }^{-1}$ de sílica pura ( $95 \%$ de $\mathrm{SiO}_{2}$ proveniente do processamento de britagem do rejeito de Quartzito), e T2: sem silício. As variáveis analisadas foram: altura de planta, diâmetro de haste, comprimento de raiz, comprimento e largura de folha, diâmetro vertical e horizontal de fruto, peso e número de frutos. Os dados foram analisados pelo teste de F com $5 \%$ de significância. Observou-se diferença significativa para altura de planta, peso de fruto e diâmetro vertical de fruto com os maiores valores médios obtidos nas plantas que receberam aplicação de silício. Conclui-se que o silício é promissor para o desenvolvimento e crescimento do pepineiro, podendo ser uma alternativa viável e sustentável para a produção de pepino e para o ambiente.

Palavras-chave: Cucumis sativus L.. Nutrição. Sustentabilidade. 


\title{
THE USE OF SILICON DERIVED FROM THE EXTRACTION OF QUARTZITE SÃO THOMÉ IN THE CUCUMBER CULTURE
}

\begin{abstract}
The silicon absorbed by plants improves their morphology and physiology, with a reduction in the rate of transpiration and gives photosynthetic efficiency to plants, is very efficient in protecting against abiotic and biotic stresses of many cultures and can confer resistance to attack by pest insects and nematodes. The silicon derived from the extraction and mineral production of quartzite in São Thomé das Letras, MG has become a waste that generates an environmental problem and its use in agriculture can be a form of sustainable. Thus, the objective of this work was to evaluate the use of silicon, derived from the extraction and mineral production of quartzite São Thomé, in the culture of cucumber. The experiment was implemented at Fazenda São Francisco de Assis, in Três Corações - MG. The design was in randomized blocks with two treatments and 10 repetitions. The treatments used were: T1: 3 ton.ha-1 of pure silica (95\% SiO2 from the crushing of Quartzite tailings), and T2: without silicon. The variables analyzed were: plant height, stem diameter, root length, leaf length and width, vertical and horizontal fruit diameter, weight and number of fruits. The data were analyzed by the $\mathrm{F}$ test with $5 \%$ significance. A significant difference was observed for plant height, fruit weight and vertical fruit diameter with the highest average values obtained in plants that received silicon application. It is concluded that silicon is promising for the development and growth of cucumber and can be a viable and sustainable alternative for cucumber production and for the environment.
\end{abstract}

Keywords: Cucumis sativus L.. Nutrition. Sustainability.

Recebido em: 21/10/2020. Aceito em: 14/01/2021. 


\section{INTRODUÇÃO}

Apesar do silício (Si) não se enquadrar nos critérios de essencialidade, é um elemento que pode ser considerado importante para o desenvolvimento de muitas culturas agrícolas (EPSTEIN; BLONN, 2005; GONZÁLEZ; CEBALLOS; BENAVIDES, 2015), pois proporciona grandes benefícios às plantas, tais como: diminuição do acamamento, estímulo ao crescimento, à produção e à proteção das plantas (PRADO, 2000).

Esses benefícios são mais evidentes e potencializados em plantas consideradas acumuladoras de $\mathrm{Si}$, aquelas que possuem teor foliar acima de $1 \%$. As plantas não acumuladoras possuem teor de silício menor que 0,5\%. A cucurbitácea, Cucumis sativus L, é considerada uma cultura intermediária quanto ao acúmulo de Si, com teor de sílica de 0,5 a 1 g.kg-1 (MA; MIYAKE; TAKAHASHI, 2001). Em geral as monocotiledôneas acumulam mais Si do que as dicotiledôneas, diferença que se deve mais a fatores filogenéticos que às condições ambientais (GONZÁLEZ; CEBALLOS; BENAVIDES, 2015; ZARGAR et al., 2019).

Estudos demonstram o envolvimento do silício em vários aspectos morfológicos, fisiológicos e bioquímicos da vida das plantas, com papéis diversos (LIMA et al., 2010; TUNES et al., 2014). O silício pode, por exemplo, provocar a ativação do mecanismo natural de defesa das plantas, como os compostos fenólicos como a quitina, peroxidases e acúmulo de lignina na parede celular proporcionando um papel importante nas relações planta-ambiente, pois pode dar à cultura melhores condições para suportar adversidades climáticas, edáficas e biológicas, tendo como resultado final um aumento na produção das plantas e produtos de maior qualidade (REIS et al., 2007). O Si é capaz de aumentar a resistência natural das plantas possibilitando uma agricultura mais sustentável (CAMARGO; KORNDÖRFER; PEREIRA, 2007; ZARGAR et al., 2019).

Assim a adubação com silício proporciona aumento no crescimento e na produção de muitas culturas (ALBUQUERQUE; SANTOS; FARIAS, 2014), como o pepineiro, considerada cultura intermediária em relação ao acúmulo de Si.

O pepineiro pertence à família Cucurbitaceae e está adaptado a regiões de clima tropical e com temperaturas elevadas até $30^{\circ} \mathrm{C}$ (GOTO, 2003; MARTINS et al., 2018). É uma cultura que necessita de condições de alta luminosidade ou fotoperíodo longo e solos férteis 
e bem drenados com pH em torno de 5,5 a 6,8; deve ser irrigado com frequência para que as plantas não sofram estresse hídrico.

O presente estudo representa uma alternativa ao problema de não haver área suficiente para expandir os depósitos de resíduos proveniente da extração de quartzito, e o seu aproveitamento na agricultura pode contribuir de forma significativa para a solução de questões ambientais, devido à reutilização deste mineral para nutrição de plantas e controle de pragas.

Em razão de muitas dúvidas que o agricultor possui em relação ao Si na agricultura e na produção de pepineiro, objetivou-se com o presente estudo avaliar o uso desse mineral no desenvolvimento e crescimento desta cultura, e principalmente encontrar soluções sustentáveis de aproveitamento de rejeito originário da extração e comercialização de quartzito São Thomé composto por 95\% de Si.

\section{MATERIAL E MÉTODOS}

O experimento foi instalado na Fazenda São Francisco de Assis no município de Três Corações/MG - Brasil. A região possui clima tropical de altitude predominante nos planaltos e serras do sudeste brasileiro, com altitude aproximada de 800 a 900 m, com precipitação e temperatura média anual de $1401 \mathrm{~mm}$ e 20,2ㅇ C respectivamente (IBGE, 2014).

Anteriormente à instalação do experimento foi realizada análise de solo (Tabela 1).

Tabela 1 - Análise de solo da Fazenda São Francisco De Assis - Três Corações - MG - (Labominas - Laboratório Agronômico)

\begin{tabular}{clll}
\hline Elementos & Resultado & Interpretação & Val. Referência \\
\hline $\mathrm{pH}$ & 5,4 & Médio & Ideal $(5,5$ a 6,6$)$ \\
\hline $\mathrm{V} \%$ & $39 \%$ & Baixo & Ideal $(70-80 \%)$ \\
\hline $\mathrm{Ca}$ & $31 \%$ & Baixo & Ideal $(60$ a $70 \%)$ \\
\hline $\mathrm{Mg}$ & $7 \%$ & Baixo & Ideal $(10$ a $20 \%)$ \\
\hline P- disponível & $2,2 \mathrm{mg} / \mathrm{L}$ & Muito Baixo & Ideal $\left(>9 \mathrm{mg} / \mathrm{dm}^{3}\right)$ \\
\hline
\end{tabular}

Fonte: os autores.

O plantio do pepino foi realizado manualmente e em sulcos, na direção leste - oeste, pois o pepino necessita de condições de alta luminosidade ou com luz solar direta por algumas 
horas por dia. 0 espaçamento utilizado foi de 0,45 $\mathrm{m}$ entre plantas e 0,60 $\mathrm{m}$ entre linhas, compreendendo área total de $151,2 \mathrm{~m}^{2}$, e cada parcela com dimensão de $3,15 \mathrm{~m}$ de comprimento e 2,4 m de largura, totalizando 7,56 $\mathrm{m}^{2} /$ parcela.

Para o preparo do solo primeiramente foi incorporada a adubação orgânica com 10,58 ton.ha-1 ${ }^{-1}$ de esterco bovino curtido, o que correspondeu a $8 \mathrm{~kg} /$ parcela, incorporados manualmente a $10 \mathrm{~cm}$ de profundidade 30 dias antes do plantio em todas as parcelas.

Para a incorporação do silício ao solo foi realizada sua distribuição aos 14 dias antes do plantio. A sílica utilizada no experimento foi Sílica pura $\mathrm{SiO}_{2}$ com $95 \%$ de pureza com granulometria ou malha de 400 mm, adquiridas da Empresa de Extração de Pedras São Thomé Ltda, que para esta empresa e demais empresas mineradoras é um problema ambiental.

As sementes de pepino, cv. Caipira, obtidas no comércio local, foram semeadas em tubetes com substrato comercial. Foram três sementes por tubete e após emergência foi realizado o desbaste deixando-se a muda mais vigorosa.

O plantio e os tratos culturais foram realizados seguindo as recomendações para a cultura obtidas em Sediyama et al. (2012), como: desbaste, tutoramento, controle de plantas espontâneas com capina manual e irrigação por aspersão todos os dias no final da tarde até o final do ciclo da cultura.

O delineamento experimental foi realizado em blocos casualizados (DBC), com 10 blocos e 2 parcelas por/bloco.

Foram avaliados dois tratamentos sendo: T1: Sílica pura $\left(95 \% \mathrm{SiO}_{2}\right)$ incorporada na dosagem referente a 3 ton.ha-1 ou 2,26 kg por parcela e T2: sem aplicação de sílica.

No final do experimento, aos 90 dias do plantio, em três plantas por parcela, escolhidas ao acaso, foram avaliadas as variáveis altura de planta $(\mathrm{m})$, diâmetro de haste $(\mathrm{mm})$, comprimento de raiz de planta $(\mathrm{cm})$, largura e comprimento de folhas $(\mathrm{cm})$, diâmetro horizontal e vertical de frutos $(\mathrm{cm})$, número e peso $(\mathrm{Kg})$ de frutos. Para a medição do diâmetro foi utilizado paquímetro, e fita métrica para medir a altura de planta e comprimento de raiz. Para o peso de frutos foi utilizada balança manual digital.

Foi realizada a análise de dados pelo teste de $\mathrm{F}$ a $5 \%$ de significância com auxílio do sistema computacional SISVAR versão 5.2 (FERREIRA, 2011). 


\section{RESULTADOS E DISCUSSÃO}

A partir dos resultados obtidos observou-se resposta positiva à adubação com silício, obtendo-se maiores valores para altura de planta, diâmetro vertical de fruto e peso médio de frutos de pepineiro em comparação às plantas que não receberam adubação com silício (TABELA 2 e 3).

Tabela 2 - Altura de planta, diâmetro de haste, comprimento de raiz, comprimento e largura de folha de pepineiro com e sem silício. Fazenda São Francisco De Assis - Três Corações, MG

\begin{tabular}{cccccc}
\hline Tratamento & $\begin{array}{c}\text { Altura de } \\
\text { planta } \\
(\mathrm{m})^{*}\end{array}$ & $\begin{array}{c}\text { Diâmetro } \\
\text { de haste } \\
(\mathrm{mm})^{\mathrm{ns}}\end{array}$ & $\begin{array}{c}\text { Comprimento de } \\
\text { raiz }(\mathrm{cm})^{\mathrm{ns}}\end{array}$ & $\begin{array}{c}\text { Comprimento de } \\
\text { folha }(\mathrm{cm})^{\mathrm{ns}}\end{array}$ & $\begin{array}{c}\text { Largura de } \\
\text { folha }(\mathrm{cm})^{\mathrm{ns}}\end{array}$ \\
\hline Com Si & 1,31 & 3,4 & 20,44 & 11,17 & 4,34 \\
Sem Si & 0,96 & 2,8 & 9,04 & 4,76 & 0,34370 \\
\hline$P$ & 0,00129 & 0,277723 & 0,088285 & 0,31702 & 0,83 \\
\hline
\end{tabular}

*Médias significativas pelo teste de $\mathrm{F}(p \leq 0,05)$. ${ }^{\text {ns }}$ Médias não significativas pelo teste de $\mathrm{F}(p>0,05)$. Fonte: os autores.

Tabela 3 - Diâmetro vertical e horizontal de fruto, e número médio e peso médio de fruto de pepineiro com e sem silício. Fazenda São Francisco De Assis - Três Corações, MG

\begin{tabular}{ccccc}
\hline Tratamento & $\begin{array}{c}\text { Diâmetro horizontal de } \\
\text { fruto }(\mathrm{cm})^{\mathrm{ns}}\end{array}$ & $\begin{array}{c}\text { Diâmetro vertical } \\
\text { de fruto }(\mathrm{cm})^{*}\end{array}$ & $\begin{array}{c}\text { Número médio de } \\
\text { frutos }^{\mathrm{ns}}\end{array}$ & $\begin{array}{c}\text { Peso médio } \\
\text { de frutos } \\
(\mathrm{kg})^{*}\end{array}$ \\
\hline Com Si & 20,94 & 7,94 & 39,1 & 17,8 \\
Sem Si & 11,72 & 4,85 & 22,6 & 4,26 \\
\hline$P$ & 0,109837 & 0,02044 & 0,17757 & 0,023675
\end{tabular}

*Médias significativas pelo teste de $\mathrm{F}(p \leq 0,05)$. ${ }^{\text {ns }}$ Médias não significativas pelo teste de $\mathrm{F}(p>0,05)$. Fonte: os autores.

No entanto, as variáveis diâmetro de haste, comprimento de raiz e de folha, largura de folha, diâmetro horizontal e número médio de frutos, de plantas de pepino que receberam silício não apresentaram diferença significativa em comparação às plantas sem silício.

Silva (2016) ao realizar fertilização foliar com silicato de potássio também verificou incremento na altura das plantas de pepino como no presente trabalho. No entanto, não 
detectou diferença significativa para peso de fruto, diâmetro horizontal e vertical, provavelmente por utilizar silício foliar e não via solo, pois as plantas possuem metabolismo diferente para absorção de nutrientes.

Miyake e Takahashi (1982) também reportaram que a aplicação de silício provocou aumento significativo na altura de planta e no peso de frutos de pepinos. Contudo Santos et al. (2018) verificaram que plantas de pepineiro tiveram o desenvolvimento afetado por diferentes doses de silicato de cálcio e magnésio.

Em trigo, a adubação silicatada via solo também foi responsável pelo aumento no número de sementes, peso e no rendimento de sementes, além de manter a qualidade fisiológica das sementes produzidas (TAVARES et al., 2014). No entanto, para a cultura do milho a adubação silicatada via foliar não afetou significativamente a altura de plantas e o diâmetro do colmo (TEODORO et al., 2014).

A diferença na altura de planta e diâmetro vertical de fruto pode também ser explicada pelo acúmulo de silício nas células da epiderme, provocando à ativação da lignina, melhorando a rigidez das folhas, a absorção dos fotossintetizados e a translocação destes nutrientes para a parte aérea das plantas e também nos frutos, tornando - os mais suculentos e com maior desenvolvimento (ETESAMI; JEONG, 2018).

O comprimento total e número total de folhas, como também a matéria seca da parte aérea e matéria seca total de plantas de pepineiro apresentaram aumento significativo quando receberam tratamento com silício em comparação com outros produtos (BELAN et al., 2013).

Folhas de alface foram beneficiadas com a aplicação de silício, que proporcionou firmeza e turgidez aumentando a vida útil em 16 dias (GALATI et al., 2015).

Segundo os estudos de Lima Filho, Lima e Tsai (1999) as folhas de pepineiros com teor alto de Si apresentam um aumento significativo na atividade de RuBP-carboxilase melhorando a reação de conversão de dióxido de carbono em proteína solúvel, melhorando a parte morfológica das plantas de pepineiro explicando o aumento nas diferenças encontradas neste experimento.

As plantas de pepino do presente estudo não apresentaram dano significativo decorrente do ataque de insetos-praga e doenças, mas o efeito do silício no aumento da resistência de plantas a insetos e patógenos também pode ser observado em algumas plantas, 
como verificado nos trabalhos de Lima et al. (2010); Santos et al. (2010); Belan et al. (2013) e Santos et al. (2018). Por exemplo, pepineiros infectados com Sphaerotheca fuliginea, transferidos para um meio contendo Si, apresentaram uma rápida silicificação do tecido foliar, principalmente nas bases dos tricomas, e ao redor dos pontos de infecção, aumentando a resistência ao patógeno (SAMUELS et al., 1991; LIMA FILHO; LIMA; TSAI, 1999).

Em pepineiro Correa et al. (2005) verificaram efeitos negativos com a aplicação de Si no desenvolvimento e na população de mosca-branca, sendo apresentado como um produto alternativo a ser utilizado no manejo integrado de insetos-pragas de muitas outras culturas (PINTO et al., 2012; AMADO; RIZENTAL, 2015; MONTES; MONTES; RAGA, 2015; CAMARGO; 2016).

Os fertilizantes silicatados, do ponto de vista econômico, assumem 10 a $20 \%$ do custo de outros fertilizantes sendo relativamente acessível (FENG, 2000). Etesami e Jeong (2018) consideraram vital utilizar fertilizantes de Si na adubação de plantas.

O Si usado na adubação de plantas pode ser de fontes orgânicas como biocarvão, cinzas de cascas de arroz e compostos de esterco de gado e inorgânicos como a volastonite, sílica gel, escória rica em si, ácido silícico, silicato de sódio, silicato de potássio, silicato de cálcio, silicato de cálcio escória, sílica amorfa, etc. em áreas com falta de silício para que experimentar seus lucros econômicos e ecológicos. A aplicação de Si pode ser uma das vias disponíveis para melhorar o crescimento e produção de culturas (MEENA et al., 2014). O Si dessa forma pode ser considerado um fertilizante de alta qualidade, podendo ser usado numa agronomia economicamente correta (ZHU; GONG, 2014; ETESAMI; JEONG, 2018).

O silício proveniente das pedras de quartizito proporcionou um incremento em aspectos morfológicos de frutos de pepino (altura de planta, diâmetro vertical dos frutos e peso médio de fruto), sinalizando que o uso como adicional na nutrição de plantas poderá ser realizado como alternativa no incremento na produção de plantas, e em especial aquelas acumuladoras de silício a as intermediárias como o pepineiro.

\section{CONCLUSÃO}

O silício influenciou a altura, o diâmetro vertical e peso de frutos de pepineiro, sendo considerado promissor para o desenvolvimento e crescimento desta e de outras culturas, contribuindo também para uma agricultura mais sustentável, através da reutilização de 
resíduos do beneficiamento de minerais prejudiciais ao meio ambiente com teores de silício bastante elevado, chegando a $95 \%$ de pureza, além de proporcionar um custo benefício a mais para os produtores e mineradores de quartzito São Thomé.

\section{REFERÊNCIAS}

ALBUQUERQUE, ABEL W. de; SANTOS, J. M. dos; FARIAS, A. P. de. Produtividade e qualidade pós-colheita de Helicônia Golden Torch submetida a fontes e doses de silício. Revista Brasileira Engenharia Agrícola e Ambiental, v. 18, n. 2, p. 173-179, 2014.

AMADO, D.; RIZENTAL, M. Silício como indutor de resistência a Aphis gossypii (Hemiptera: Aphididae) e Bemisia tabaci (Hemiptera: Aleyrodidae) em algodoeiro. Conection Line, n. 12, 2015.

BELAN, L. et al. Manejo alternativo do oídio na cultura do pepino em ambiente protegido. Revista Acadêmica, Curitiba, v. 11, Supl. 2, p. S03-S112, 2013.

CAMARGO, M. S. de. Efeito do silício na tolerância das plantas aos estresses bióticos e abióticos. Informações agronômicas, n. 155, p. 1-8, 2016.

CAMARGO, M. S.; KORNDÖRfER, G. H.; PEREIRA, H. S. Solubilidade do silício em solos: Influência do calcário e ácido silícico aplicado. Bragantia, Campinas, v. 66, p. 637-647, 2007.

EPSTEIN, E.; BLOOM, A. J. Mineral nutrition of plants: principles and perspectives. Sunderland: Sinauer Associates, 2005.

ETESAMI, H., JEONG, B. R. Silicon (Si): Review and future prospects on the action mechanisms in alleviating biotic and abiotic stresses in plants. Ecotoxicology and Environmental Safety, v. 147, p. 881-896, 2018.

FERREIRA, D. F. Sisvar: a computer statistical analysis system. Ciência e Agrotecnologia (UFLA), Lavras, v. 35, n. 6, p. 1039-1042, 2011.

GALATI, V. C. et al. Aplicação de silício, em hidroponia, na conservação pós-colheita de alface americana 'Lucy Brown' minimamente processada. Ciência Rural, Santa Maria, v. 45, n. 11, p. 1932-1938, nov./2015.

GONZÁLEZ E. M.; CEBALLOS, J. M.; BENAVIDES, O. B. Producción de forraje verde hidropónico de maiz Zea mays. L. en invernadero con diferentes niveles de silício. Revista de ciências agrícolas, v. 32, n. 1 p. $75-83,2015$.

GOTO, R. Pepino: Cucumis sativus L.: Programa brasileiro para a modernização da horticultura. São Paulo: CQH/CEAGESP, 2003. Normas de classificação do pepino. Disponível em: http://www.ceagesp.gov.br/wp-content/uploads/2015/07/pepino.pdf. Acesso em: 16 jan. 2018. 
LIMA FILHO, O. F. de; LIMA, M. T. G. de; TSAI, S. M. O silício na agricultura. Informações Agronômicas, Piracicaba, n. 87, p. 1-7, 1999. (Encarte técnico, 132).

LIMA, et al. Quantificação da ferrugem asiática e aspectos nutricionais de soja suprida com silício em solução nutritiva. Summa Phytopathologica, v. 36, n. 1, p. 51-56, 2010.

MA, J. F.; MIYAKE, Y., TAKAHASHI, E. Silicon as a beneficial element for crop plant. In: DATNOFF, L.E.; SNYDER, G.H., KORNDÖRFER, G.H. (ed.). Silicon in agriculture. Amsterdam: Elsevier Science, 2001. p. 17-39.

MARTINS, J. K. D. et al. Avaliação da produtividade do pepino conduzido em sistemas de tutoramento horizontal e vertical com e sem consórcio com amendoim em Rondônia. Enciclopédia Biosfera, Goiânia, v. 15 n. 27, p. 151, 2018.

MEENA, V. et al. A case for silicon fertilization to improve crop yields in tropical soils. Proceedings of the National Academy of Sciences, India: Section B: Biological Sciences, v. 84, n. 3, p.505-518, 2014.

MIYAKE, Y.; TAKAHASHI, E. Effect of silicon on the growth of cucumber plants in a solution culture. Journal Soil Science Plant Nutrition, v.53, p. 15-22, 1982.

MONTES R. M., MONTES, S. M. N. M.; RAGA, A. O uso do silício no manejo de pragas. São Paulo: Instituto Biológico-APTA, 2015. Documento Técnico, n. 17, p. 1-13.

PINTO, D. G. et al. Alterações fisiológicas após aplicação de silício em cacau e sua influência na preferência por pulgões. Revista Ceres, Viçosa, MG, v. 59, n. 3, p. 360-367, 2012.

PRADO, R. M. Resposta da cana-de-açúcar à aplicação de escória silicatada como corretivo de acidez do solo. 2000. Dissertação (Mestrado em Agronomia) - Faculdade de Engenharia da Universidade Estadual Paulista, Ilha Solteira, 2000.

REIS, T. H. P. et al. O silício na nutrição e defesa de plantas. Boletim Técnico, v. 82, Belo Horizonte, 2007.

CORREA, R. S. B. et al. Silicon and acibenzolar-s-methyl as resistance iducers in cucumber, against the whitefly Bemisia tabaci (Gennadius) (Hemiptera: Aleyrodidae) biotype B. Neotropical Entomology, v. 34, n. 3, p.v429-433, 2005.

SAMUELS, G. J. et al. A synopsis of Nectria subg Dialonectria. Mycological Papers, v. 164, p. 147, 1991.

SANTOS, B. H. C. dos et al. Silicato de cálcio e magnésio no controle de Meloidogyne javanica em pepineiro em diferentes texturas de solo. Revista Brasileira de Agropecuária Sustentável, v.8, n. 1, p. 104-109, mar. 2018. 
SEDIYAMA, M. A. N. et al. Manejo da poda em plantas de pepino tipos Aodai, Japonês e Caipira. Horticultura brasileira, v. 30, n. 2, jul. 2012.

SILVA, A. M. Q. Respuesta a las aplicaciones de silicio en el cultivo de pepino (Cucumis sativus L) variedad modan, en condiciones de estrés hídrico bajo cubierta en culiacán, sinaloa. 2016. Tesis (Engenharia Agrônoma) - Universidad de Ciencias Aplicadas y Ambientales U.D.C.A, Facultad de Ingenierías, Bogotá, 2016.

TAVARES, L. C. et al. Adubação silicatada em trigo: qualidade e rendimento de sementes. Revista Faculdade Agronomica, v. 113, n. 1, p. 94-99, 2014.

TEODORO, P. E. et al. Desempenho de híbridos de milho sob aplicação foliar de Silício no cerrado sul-mato-grossense. Bioscince journal, Uberlandia, v. 30, supplement 1, p. 224-231, 2014.

TUNES, L. V. M. de et al. Qualidade fisiológica, sanitária e enzimática de sementes de arroz irrigado recobertas com silício. Revista Ceres, Viçosa, MG, v. 61, n.5, p. 675-685, set/out, 2014.

ZARGAR, S. M. et al. Role of silicon in plant stress tolerance: opportunities to achieve a sustainable cropping system. Biotech, v. 9, n. 73, 2019.

ZHU, Y., GONG, H. Beneficial effects of silicon on salt and drought tolerance in plants. Agronomy for Sustainable Development, v. 34, p. 455-472, 2014. 